This is the peer reviewed version of the following article: Vallée-Tourangeau, Freédéric and March, Paul L. (2020) Insight out : making creativity visible. The Journal of Creative Behavior, 54(4), pp. 824-842., which has been published in final form at https://doi.org/10.1002/jocb.409. This article may be used for non-commercial purposes in accordance with Wiley Terms and Conditions for Use of Self-Archived Versions. 


\title{
Insight Out: Making Creativity Visible
}

\author{
Frédéric Vallée-Tourangeau \\ Kingston University \\ and \\ Paul March \\ University of Oxford
}

\begin{abstract}
Author Note
Address correspondence to either Frédéric Vallée-Tourangeau, Department of Psychology, Kingston University, Kingston upon Thames, Surrey, UNITED KINGDOM, KT1 2EE, f.vallee-tourangeau@kingston.ac.uk or Paul March, Keble College, University of Oxford, Oxford, UNITED KINGDOM, OX1 3PG, paul.march@,keble.ox.ac.uk. We thank Wendy Ross, Gaëlle Vallée-Tourangeau and three anonymous reviewers for their thoughtful comments on a previous version of this manuscript.
\end{abstract}




\begin{abstract}
Models of creative problem solving are predicated upon mental states to explain everything from the outcome of problem-solving experiments to the emergence of artistic creativity. We present two converging perspectives that describe a profoundly different ontological description of creativity. Our analysis proceeds from a distinction between firstorder problem solving, where the agent interacts with a physical model of the problem and second-order problem solving, where the agent must cogitate a solution to a problem that is presented as a verbal description of a state of the world but where the agent does not or cannot transform physical elements of a problem. We acknowledge the recent evidence that foregrounds the importance of working memory in problem solving, including insight problem solving. However, we stress that the impressive psychometric success is obtained with a methodology that only measures second-order problem solving; we question whether first-order problem solving is equally well predicted by measures of cognitive or dispositional capacities. We propose that if mental simulation is replaced by the opportunity to engage with a physical model of a problem then the environment can provide affordances that help the participant to solve problems. In the second part of the paper, we present the subjective experience of an artist as he monitors the micro-decisions that occur during the morphogenesis of a large, clay, sculptural installation. The testimony is a vivid demonstration that creative action occurs, not in the brain, but in the movement between the hand and the clay. Insight becomes outsight.
\end{abstract}




\section{Insight Out: Making Creativity Visible}

It would be difficult to overstate the importance and influence of Wolgang Köhler's (1925) "The mentality of apes" on the psychology of problem solving, insight and creativity. As a nascent scientific discipline, psychology was embracing behaviourism with its associated methodological and ontological proclivities, yet Köhler's ethological observations and inferences were couched in mentalist terms. Problem solving in these chimpanzees was not described in terms of the concatenation of stimulus-response sequences through gradual associative learning. Rather, some of the solutions to the problems engineered by Köhler for these animals appear to reflect insight, a sudden discovery of a path to solution. In one instance, Sultan - one of Kohler's star performers, was shown a banana attached to the roof of an enclosure, 2 meters from the ground. A box is placed in the middle of the room; the banana is 2.5 meters from the box. Köhler writes (p. 41): "Sultan suddenly stood still in front of the box, seized it, tipped it hastily straight towards the objective, climbed upon the box and springing upwards with all his force, tore down the banana." In what would be called the Gestalt account of insight, a solution reflects the sudden reconfiguration of perceptual elements. The mental representation of the problem is initially misaligned with the representation of the goal. The tension between these mental representations trigger unconscious processes that seek to harmonize these two representations, to create a good gestalt (Gilhooly \& Webb, 2018).

The current science of insight problem solving in particular shares a lot in common with this initial theoretical account. Animal ethology no longer prefigures so centrally of course, and an experimental methodology is largely favoured over single case studies, but the aim is to identify the cognitive processes that turn an initially incorrect or unproductive problem representation into one within which the solution offers itself in the agent's mental look ahead horizon. To forego the cost and patience involved in observing people's problem 
solving outside the lab, cognitive psychologists create miniature problem solving environments designed to encourage an incorrect representation of an ostensibly simple problem or riddle. In the psychologist's laboratory, insight problem solving research usually proceeds by presenting participants a riddle, such as "the thing that can move heavy logs, but cannot move a small nail" (Luo \& Nikki, 2003, p. 317), or how do you throw a ping pong ball in such a way that it travels a certain distance, comes to a dead stop and then reverses direction (to adapt, Ansburg \& Dominowski, 1980) or "if you have black socks and brown socks in your drawer mixed in the ration of $4: 5$, how many socks do you need to take out to be sure of having a pair of the same color" (Fleck \& Weisberg, 2013, p. 446; this problem is also used in Chuderski \& Jastrzębski, 2018, reviewed below). These riddles are created to mislead, to encourage an incorrect interpretation that will frustrate the direct application of long term memory knowledge to identify a solution. To create a conceptual impasse is the point, of course, and then researchers observe how the impasse is overcome. A few participants will eventually say, 'river', 'vertically', and '3 socks', but most will labour fruitlessly until the end of the allocated time (usually a few minutes). Using a broad range of measures, psychologists are interested in the phenomenology of insight, neuroimages of areas that are more active when an insightful solution is achieved, or the conscious analytic strategy revealed through protocol analysis.

The current debate in the psychology of insight problem solving (e.g., Gilhooly \& Webb, 2018) pitches the so-called business-as-usual view against the special-processes view. The latter has roots in early Gestalt ideas: insight is the result of a swift change in the manner with which a problem is represented in the mind. The sudden awareness of the solution suggests that insight is not the product of a conscious deliberate analysis of the problem, proceeding incrementally, helping the agent formulate a workable solution gradually over time. The 'special' in special processes underscores that insight is the product of non-routine cognition that largely operates non-consciously (Ohlsson, 2018). If routine cognition, in turn, 
is in the business of helping an agent to plan and solve problems, then the business-as-usual view holds that insight is the product of conscious, deliberate, and incremental effort to solve a problem. From this perspective, a breakthrough may well yield a eureka moment, but that distinct phenomenological signature does not imply that something other than routine cognition is involved in insight.

\section{Working Memory: The Psychometric Approach}

If insight is the result of unconscious thinking, then working memory, as the conscious mental space over which people mentally manipulate, rehearse, and store relevant information when thinking, might not be implicated in insight problem solving. In turn, the business-as-usual view holds that a participant's working memory capacity and executive function skills should predict that participant's ability to solve insight problems. Researchers employ a psychometric approach to test these ideas (Chuderski \& Jastrzębski, 2018): participants sit through a session composed of insight problems and various working memory capacity tests. Performance on these insight problems - usually measured simply in terms of the number of correct answers - is correlated with performance on working memory tests. For example, Gilhooly and Fioratou (2009) reported that measures of verbal and visuo-spatial working memory explained a significant portion of variance in insight problem solving. This suggests that people's ability to solve the riddles that are offered as insight problems, correlate with working memory capacity, which in turn correlates with measures of intelligence. The state of the art of this approach is well illustrated in Chuderski and Jastrzębski (2018): A large and heterogeneous sample of participants were tested over an 8hour session on a large number of different insight problems (verbal, spatial, mathematical and matchstick arithmetic problems as well as compound remote associates presented as a pen and paper exercise) and a wide set of working memory and executive function tasks; participants also completed as a battery of personality, anxiety and motivation measures. Scores on all these measures are, in the first instance, correlated, yielding a sizable 
correlation matrix (Table 5, p. 270); measures of working memory and executive function all correlated positively with composite performance scores for all types of insight problems. Factor analysis is then used to identify and confirm the presence of a separate reasoning ability and insight ability latent variable, themselves strongly correlated and significantly linked to all measures of working memory capacity and executive function skills (Figure 3, p. 272). Chuderski and Jastrzębski (2018, p. 276): conclude: "Above half of the variance in insight problem solving explained here by working memory capacity constitutes a strong refutation of the claims (...) that working memory is unrelated, or even negatively related, to insight problem solving”. The psychometric approach thus foregrounds the importance of working memory capacity in insight ${ }^{1}$ problem solving. Such a model explains creative problem solving in terms of an agent's cognitive capacities: The better these capacities the better positioned an agent is at solving problems.

Let's consider how the research on insight problem solving proceeds in the laboratory and how the research methodology employed by the psychometric approach validates and reinforces a model of problem solving that casts working memory as playing a central role. The dominant research paradigm is erected on the twin pillars of mentalism and methodological individualism. The research proceeds on the assumption that the world is mentally represented and located inside the person, or more specifically, the person's head. As a result of this ontological position, the research methodology must focus on the individual and his or her internal cognitive capacities. The individual's mental representations are transformed on the basis of rules and operators. It is not surprising that individual differences in working memory capacity and intelligence explain a substantial portion of the variance in problem solving performance: Working memory capacity underlie

\footnotetext{
${ }^{1}$ Classifying problems as 'insight' problems does not mean or guarantee a priori that a solution for these problems will exhibit the key phenomenological elements of an insightful one (viz. suddenness, positive emotionality, conviction of the correct solution; Bowden \& Grunewald, 2018).
} 
a person's ability to construct, maintain and transform representations of the world. Crucially, the methodology employed tasks participants to think about short vignettes - a few words or sentences - that describe ambiguously some state of the world; in other words, participants are not embedded in a physical world to solve a problem that arises in that world, (first order problem solving), but rather problem solving is removed from the external world, conducted on the basis of representations of the world (second-order problem solving). Second order problem solving proceeds from participants' interpretation and representations of these representations. In other words, the problems that could arise in a physical world, corresponding to physical processes of varying complexity, are presented as second-order abstractions. First-order problem solving is impossible since participants cannot engage or interact with a physical presentation of the problem. Second order problem solving carries with it a representational toll and as a result, individual differences in the ability to maintain and transform mental representations — as gauged in terms of working memory capacitycorrelate with problem solving performance. The research methodology thus validates rather than tests the foundational pillars of the psychometric research programme.

First-order Problem Solving: Insight Distilled through Action. Let's transpose the socks problem, as described above, from verbal riddle into a physical model of the problem (from second order to first order). Imagine a duffle bag with 40 black socks and 50 brown socks. Our participant reads the problem description and is invited to determine how many socks she'll have to sample before getting a pair of matching colour. She's told she can dig into the bag and pull a few socks, one at a time, to help her solve the problem. The misleading ratio information in the problem description might not attract her attention as much as it would otherwise were she only presented with the riddle without a physical model of the problem. She might not know how to solve the problem; she starts pulling a few individual socks, not strategically, not with a plan in mind, no proto-solution guiding her action, but simply exploring, interacting with the problem and observing results. The 
misleading ratio information quickly fails to exert any attraction; rather she's looking at the results of her sampling from the bag. She may pull two black socks from the start, tempted to say that the answer might simply be 'two', but realises that she's been lucky, pulls a third one and fourth one, and the solution dawns on her; the solution is distilled through action and results. The insight, if there is one to experience, takes place when she observes the results of her actions.

We offer this intuition pump as a means to illustrate the plausibility of how the solution to the problem might unfold on the basis of actions. Pulling socks out of the bag is an epistemic action (e.g., Kirsh, 2009), each drawn sock providing information and the outcome of a few draws brings the solution into focus. That is, the solution of the problem is observed rather than mentally simulated. No exact plan or arithmetic knowledge determines her behaviour. Interacting with a physical model of the problem offers perceptual information and action possibilities. Problem solving is taken out of the head and enacted in the world: The solution is physically crafted and perceived. First-order problem solving is generally embodied and necessarily situated, that is reflecting the assembly of a "transient extended cognitive system" (Wilson \& Clark, 2009) that recruits and exploits artifacts in the reasoner's immediate environment. However, the more important aspect of first-order problem solving may be interactivity, that is how a new idea, the discovery of a solution in this instance, is distilled through actions and observations of their resulting impact on the environment. Firstorder problem solving unfolds contingently along a spatio-temporal trajectory, shaped by actions and dotted with preliminary observations that cue additional actions, and this recursive process may result in the discovery of a solution (Vallée-Tourangeau \& ValléeTourangeau, 2014). It is incremental, and in that respect shares similarities with the gradualism espoused by Weisberg (2018; we return to this point below).

The reasoner, the physical reality of the problem, and the action possibilities offered by the external environment, configure a cognitive ecosystem (Hutchins, 2010). From a 
cognitive ecology perspective, to adapt Hutchins (2010, p. 712) "perception, action, and thought are inextricably integrated: cognitive activity is profoundly situated, social, embodied, and richly multimodal". Interactivity assembles, creates and maintains this ecosystem. Furthermore, it might be more useful to profile the cognitive resources of the system as a whole, rather than those of the agent segregated from the system. For example, consider a simple mental arithmetic task, consisting of adding a series of single-digit number. If the numbers appear on tokens that the agent can touch and move, then the transient extended cognitive system in this instance will scaffold the working memory resources of the agent, may improve executive skills, attenuate the negative impact of maths anxiety on performance, and permit the expression of more complex arithmetic strategies (ValléeTourangeau, 2013; Vallée-Tourangeau, Sirota, \& Vallée-Tourangeau, 2016).

In first-order problem solving, interim results, that is proto-solutions that correspond to intermediary changes in the physical problem presentation, emerge over time and space. The processes at play operate on different time scales: At very short time scales, perception-action loops chart the problem-solving trajectory, at slower time scales the reasoner reflects on results, taking stock of the past and formulating hypotheses as to what should be done next. The problem-solving trajectory is paved through dynamic changes within the physical configuration of the problem, which in turn creates a shifting topography of action affordances. These actions need not be — and at very short time scales, cannot be - mediated by complex representations of the problem. The physical world is there to experience and actions may reflect the perceptual exploration of the problem. Traditional concerns with working memory capacity make sense only if problem solving is assumed to involve a hefty representational burden, that is if it is primarily a second-order problem solving activity. In contrast, if problem solving proceeds primarily on the basis of actions that modify the physical substrate of the cognitive ecosystem, then changes can in turn focus attention and cue action. The solution emerges through changes in the problem configuration enacted and 
observed by the reasoner. As a process that extends over time and space, problem solving follows a contingent itinerary which becomes important to record if we want to understand how a problem is actually solved.

How first-order problem solving can address the current debate in insight problem solving researcher, pitting special processes and business-as-usual accounts, remains to be systematically explored. The process of undergoing and transformation in first order problem solving shares some similarities with the gradualist position developed by Weisberg across papers that span nearly half a century (e.g., Weisberg \& Suls, 1973; Weisberg, 2018). Here innovative thinking, problem solving and creativity are cast in terms of analytic processes driven by local analogies with past ideas and practice: There might be important discontinuities reflected in a finished work of art (say) with what was done before, but these discontinuities were the result of a temporarily-extended process of small changes. Weisberg aggregates evidence for this position from a wide range of methodologies, including experiments, verbal protocol analysis and the re-analysis of innovations cast by some as involving sudden insight in terms of a conjectured set of inferences based on local analogies and prior knowledge (e.g., da Vinci's aerial screw). Perhaps closer still to the position we advocate here is the notion of 'data-driven' restructuring in insight problem solving offered in Fleck and Weisberg (2013): By this they mean that restructuring occurs in response to an observed change in the environment caused by the reasoning agent or capitalizing on a serendipitous re-arrangement produced by extraneous causes. It is worth noting that in Fleck and Weisberg (2013), data-driven restructuring is more likely to occur with problems presented to participants that involve the manipulation of actual artefacts (e.g., the triangle of coins problem; Vallée-Tourangeau, 2014).

\section{First Order Problem Solving in the Lab}

We recently examined how participants solved a so-called insight problem: how to distribute 17 animals in four enclosures such that each enclosure contains an odd number of 
animals. On the surface, the problem masquerades as a simple arithmetic one; however, no combination of four odd numbers can sum to 17 . The challenge then is to abandon this arithmetic interpretation and work on the spatial arrangements of the enclosures in order to exploit set intersections. In a series of experiments (Vallée-Tourangeau, Steffensen, ValléeTourangeau, \& Sirota, 2016) we observed participants working on a solution for a period of 10 minutes in one of two task environments that differed in the tools available and the type of interactivity. Participants in one environment were given an electronic tablet and a stylus with which to sketch a solution. In the first of two experiments, no participant solved the problem, in a second $17 \%$ did. With a stylus, participants are encouraged to write whole numbers - as opposed to make individuated marks corresponding to the animals - labouring an impossible arithmetic solution. Equally important, once pens were drawn, often as crosses that created quadrants on the middle of the tablet, pens were rarely re-drawn. Thus, the common pen configuration reinforced the misleading arithmetic interpretation, and their static nature suggested that they were rarely if ever the focus of attention. In sum, the tools and actions afforded by this ecosystem were more likely to perpetuate the incorrect interpretation of the problem which reinforced a stale problem-solving strategy.

In a second condition, participants were invited to build a solution of the problem with pipe cleaners and 17 animal figurines; they were not given pen or stylus, paper or electronic tablet, with which to sketch a solution. The model building environment configures a very different cognitive ecosystem. For one, participants cannot easily manipulate explicit number symbols, and as a result the quixotic iterative search of which four odd numbers can add to 17 is much less likely. Second, participants must construct pens and their activity initially focuses on the key element of the problem, namely the shape and spatial arrangements of the pens. Participants in both conditions read the same problem description, yet the different tools and action possibilities facilitate very different problem solving strategies. In the model building environment, over $40 \%$ of the participants produced a working model of the solution 
in the allocated 10 minutes with an additional $15 \%$ building proto-solutions involving intersecting enclosures. Building a model of the solution helped participants to abandon an unproductive arithmetic interpretation of problem. However, interactivity weaves a path, and the artefacts provided sometimes cued participants to perform actions that lead to a cul-desac. For example, a few built tagliatelle-type nests for the enclosures, presumably to prevent the animals from jumping over. In one of the experiments, the figurines employed were zebra-shaped paper clips, and a few participants took advantage of this affordance and clipped animals onto the pipe cleaning pieces. Building high nests and clipping animals to the perimeters of enclosures created perspectives which ruled out envisaging an overlapping set solution. The point is that a participant's problem-solving trajectory is singular and contingent. Because action possibilities depend on previous results, it becomes particularly important to examine detailed video evidence on a case by case basis, in order to understand how each participant manages to build a working model.

We profiled participants in both conditions in terms of their working memory capacity — computational and visuo-spatial span tests courtesy of the Randall Engle lab at Georgia Tech — as well as their thinking dispositions using the short version of an actively open-minded thinking scale (Haran, Ritov, \& Mellers, 2013) and the need for cognition scale (Cacioppo, Petty, \& Kao, 1984) to gauge their openness to new ideas, willingness to abandon discredited ones, and attitudes towards effortful cognition (such as enjoying challenging puzzles). Since participants were randomly allocated to either the tablet or the model building condition, we expected no difference between conditions in terms of these psychometric dimensions, and indeed there were none. Thus, participants who failed to produce a solution to the 17 animals problem had the same working memory capacity and the same thinking dispositions as those in the model building condition. In the model building condition we also compared participants who built a working model of the solution with those who failed: None of the psychometric variables predicted problem solving success. Still, these psychometric 
observations must be interpreted cautiously in light of the modest sample from which they were made.

Theoretical concepts traditionally offered to explain problem solving performance in terms of features internal to the reasoner failed to account for any of the variance in our experiment. Rather, to understand how successful problem solving performance comes about, it makes more sense to refocus research onto the cognitive ecosystem within which solutions are enacted and through which a solution-probing path is woven contingently. Insight is connected, not so much to a new way of looking at a problem, but to a new way a problem can look once its physical appearance has been shaped and reshaped through interactivity.

Yet, problem solving research in psychology proceeds under the aegis of a psychometric model that assumes problem solving to be a mental phenomenon concerned with the transformation of mental representations (e.g., representations of the problem, the initial state, the goal state). People vary in the cognitive capacities that facilitate the transformations of mental representations: A mind that is better at simultaneously holding and manipulating many elements of a problem representation will be more adept at solving problems. The psychometric model is bolstered and emboldened by correlations that evidence a link between generic cognitive capacities and problem-solving performance, whether of the insight or non-insight kind. This assumption is in turn motivated by a tacit commitment to methodological individualism: Thinking and insight are mental phenomena that require explanations in terms of mental operations that occur within an individual, or more specifically within an individual's head. As a consequence, the situated and interactive character of thinking is neglected.

The psychology of problem solving has been largely indifferent to the development of ideas in philosophy and cognitive science in terms of extended minds, situated and distributed cognition (e.g., the 4E perspective on cognition; Gallagher, 2018). Yet, beyond the methodological confines of the psychologist's laboratory, it does not require much 
ethnographic effort to notice that people think with their hands, their body, and a wide range of disparate artifacts. External resources are recruited, configuring extended cognitive systems that scaffold thinking and reasoning. Interaction with the physical environment is a key feature of thinking outside the laboratory.

Problem solving is dynamic and distributed, enacted spatio-temporarily. It is an interactive process that engages a wide range of resources, such as artefacts, people, even galaxies (e.g., as gravitational lenses). Both science and art are problem solving activities that trace paths that are deeply contingent, dotted with interim stages, proto-solutions and preliminary models. These interim products are boundary objects that bind but also transform actions and people in enacting the problem solving itinerary. To better understand the genesis of insight, of new ideas, of new forms, one should examine carefully, on the basis of detailed case studies, the conditions from which these forms emerge. In the laboratory this can be achieved only through a detailed qualitative analysis of a participant's action as he or she works within an ecosystem that affords the physical manipulation of the problem. For example, Steffensen et al. (2016) conducted a detailed analysis of the video recording (over 1200 annotations for a 10-minute session) of a single participant successfully solving the 17 animals problem. During the first two minutes, the participant spent time building enclosures. In the process, she occasionally created overlapping pens which she would promptly disassemble in order to maintain a configuration of 4 separate enclosures. The participant spent four minutes trying to distribute the 17 figurines into these separated pens in her attempts to find a solution. Frustrated with her inability to crack the problem, the participant placed all the animals in a heap in the middle of the work surface and focused her attention on the pens themselves. While fiddling with the shape of one enclosure, she accidentally created an overlap. She began to remove it but stopped just as she was about to touch one of the pens (a moment termed the 'event pivot'). Then, instead of undoing the overlap, she created another two intersections by moving the two remaining pens. With these three 
overlapping areas, she had achieved a configuration that cued some interesting possibilities for her; possibilities that were not available to her earlier in the session. Accordingly, she now proceeded to distribute the animals to match the odd number constraint.

When did she solve the problem? She solved it when she constructed a working model of the solution. Did she solve it when she produced overlapping sets? We would argue that she did not, but rather sought encouragement from this new arrangement and began systematically working at populating this new enclosure configuration. Her actions in the first 6 minutes of the session appeared to be guided by a plan that was determined by a misleading arithmetic interpretation of the problem. But her actions at the event pivot did not reflect a plan. At the point when she took advantage of an overlap, it was her actions that distilled a plan. That is, a working solution was enacted rather than mentally simulated. Her solution arises out of a contingent path that initially takes her through her failed attempts to distribute her flock into separate pens followed by an accidental overlap that was opportunistically seized and exploited to drive new distribution efforts. The event pivot at the 6-minute mark is an important discontinuity but the significance of the overlap was noticed only at a certain point in the trajectory; earlier accidental overlaps were undone. Thus, we can understand her success from a contingent-historical perspective of failures and adjustments. The physical model was constructed into a working solution, but it is incorrect to say that the construction reflected the implementation of a plan.

\section{Creative Problem Solving with Clay}

In the first part of the paper we make a distinction between first and second-order problem solving. In first-order efforts, the problem-solver comes to a solution directly by manipulating the world. In turn, second order problem solving forces the problem-solver to find a solution using mental resources alone. We have presented experimental evidence to show that, when given an option, participants change second-order situations into first-order ones and, in so doing, demonstrate more problem-solving success. However, it is the nature 
of experimental design (in which two or more variables are shown to have differential effects on a particular outcome) that it necessarily constrains explanations to those based upon linear causation. Whereas the experimental evidence we have cited shows that it is possible to construct experimental situations that are sensitive to the effects of physical interaction, the demonstrated relationship remains linear. This does not necessarily reflect the way creative problem-solving occurs in an extended, ecologically realistic situation.

The situated and distributed character of problem solving in the wild is clearly illustrated in the work of Lave (1988) and Hutchins (1995). The challenges faced by the psychometric paradigm to explain creative problem solving come sharply into focus as we scale up from experimental research in the laboratory, to problem solving in professional and creative domains of science and art. A professional creative agent (qua designer, molecular biologist, or sculptor) may toil for days, months or years on a client's brief, an engineering problem, or a large-scale clay sculptural installation. This form of creative problem solving unfolds in time and space, within a network of cultural practices - often resulting in a long trail of interim artefacts (such as sketches, models and prototypes). Clearly these agents can be profiled along psychometric dimensions, but the creative arc is complex, shaped by processes operating in different temporal dimensions; it is profoundly and unpredictably contingent.

In the second half of the paper we shift perspective and narrative voice from a nomothetic commentary on an internalist model of creative problem solving to one of the author's subjective commentary on the creative arc traced in the production of a large clay sculptural installation entitled Claustra (see Figure 1). This commentary augments and complements the critical analysis offered in the first part of the paper, but also underscores the artist as a situated agent by detailing the creative, cognitive and cultural ecosystem within which an artist enacts a work of art. The ontological position that this phenomenological approach presents can be summarized in terms of four main observations. First, a short-term 
analysis of the process of creativity tends towards a description that links an idea (cause) with an effect (art-work). This case study shows how a longer-term analysis embeds the art-work in a network of interconnecting arcs of influence, making creativity part of work-practice. Second, the chronological experience of time can be disrupted. If the development of a work of art is seen as a journey, then the journey is not always experienced chronologically - time's arrow can sometimes feel as though it is pointing backwards. Third, although cranial activity is causally associated with creative acts, the locus of creativity is not to be found in a brain isolated from its environment but in the interactive movement of hand and material. Creativity and cognition are not neural entities, they are culturally informed, subjective experiences arising out of the formation of provisional systems. Fourth, contrary to a mentalist interpretation, the experience of insight or what is known as a "lightbulb moment" is often an indication that something new, unusual and interesting has just happened in the world. It therefore seems more appropriate to replace the term 'insight' with 'outsight'2.

Insert Figure 1 here

It is clear that, as a subjective account, this commentary cannot provide an objectively valid description of the process of creativity. While accepting this shortcoming, we will return to it in our conclusions in order to make an argument for the value of such accounts. For the moment it is enough to say that we do not cite the case-study as proof for our concept of outsight. Nor do we wish to suggest that the working practice therein described is representative of artists in general. The point of the case study is to offer a systemic analysis of the process of creativity. By giving a description based upon interconnected spirals of

\footnotetext{
${ }^{2}$ Oxford Dictionary defines outsight as a "Vision or perception of external things; the capacity to see or observe; (the ability to take) an overview." We suggest reformulating "Outsight" to mean instead "arrive at an understanding or a solution of a complicated problem or situation while observing or manipulating the external world."
} 
cognitive experiences and physical transformations we hope to show that it is reasonable to reformulate the concept of insight as outsight. Rather than trying to prove the existence of outsight, our aims are more modest. We wish to show that outsight provides a plausible description of a complex, real-world example of creative problem-solving in which thinking, action and material are inseparably linked. Change the material and you change the very process of thinking itself. We wish to demonstrate that future empirical work needs to be sensitive to the transactional coupling between cognition and material.

\section{Beyond the Hylomorphic Genesis of Art}

An information processing approach to artistic creation suggests that a work of art is a material realisation of one or more ideas or internal images (e.g., Boden, 2004). This view of creative activity is called "hylomorphism" and involves the separation of things in the physical world into the matter (hyle) of which they are made and the form (morphe) such matter takes. Once form is abstracted from material it can be representationally stored. This philosophical position has a long history dating back to Aristotle. Seen like this, art has a useful representational role: It makes the internal world of the artist accessible to others. And if we wish to explore the origins of an art-work, it enables us to begin the story with the birth of a creative idea before turning a retrospective gaze on the personality of the artist and its manifestation in the artwork. This position seems reasonable. It is consistent with how art is taught in schools and it is underwritten by one of the most influential recent movements within the art world, conceptual art. It also seems to chime with subjective experience: For example, while running a clay workshop for children, one of the authors noticed how, from time to time, children as young as seven or eight years old would remain frozen in front of a lump of clay, complaining that they did not know how to start because they did not have any ideas. The children's remarks imply that the idea behind an artwork provides us with intention. Having an idea allows us to pass into action. Without an idea we do not know what we are doing and so we do nothing at all. If the activity of the brain is seen as something that 
happens separately from the activity of the world then a concept such as 'intention' becomes necessary to explain how brain activity can have an external influence (Latour, 1999). It is our contention that, by adding context, by embedding a particular creative act within an ongoing process of artistic creation, a model of creation based upon linear causation becomes impossible. Instead a more confusing, messy, systemic pattern emerges. In order to render things more comprehensible we divide the second half of the paper into four sections; we move also to a first-person account.

\section{Symmetry}

An art-work does not normally occur in isolation but as part of a process of ongoing artistic activity. Claustra was preceded by a series of sculptures (Substantia Innominata, 2014; see Figure 2). Each sculpture was both highly structured and yet perceptually indeterminate, giving the impression that there was something to recognise without the sculpture ever settling into an acceptable thing in the eye of the beholder.

Insert Figure 2 here

While making these pieces, symmetry emerged as a way of giving structure to otherwise indeterminable shapes. In addition, I found I could extend the uncertainty about my relationship with these emerging forms and their relationship with each other by creating each half, side by side, only bringing them together at the end. Working this way, I noticed that the halves had a very different relationship with each other as separate pieces compared with when they were unified. In particular, separately they created a space between them that became a quasi-thing in itself (see Casati \& Varzi, 1994, for a description of the object-status of holes). As the series progressed, I found it increasingly difficult to bring the pieces 
together. They seemed to prefer to be separate. Perhaps this resistance marks the beginnings of Claustra ${ }^{3}$.

Perceiving the space between the symmetrical parts as a quasi-thing was a discovery that I made while observing the changing juxtaposition of the parts themselves. There was no pre-existing idea or concept that I wished to make manifest. It manifested itself to me. The change of mind emerges from the repetitive rhythm of action and reaction. The separated symmetry of Claustra is ontogenetically continuous, an example of what Marion Milner (1950) calls “contemplative action". When the nascent Claustra is experienced as a change in work-practice rather than as a creative idea, I become a participant-observer in an ongoing reconfiguration of my surroundings. It feels like I am part of an evolving collaboration. But after the event, when I am not longer making, it becomes more of a struggle to describe things in these terms. In the absence of clay as a dynamic, material substrate, the memory of creative change becomes isolated and abstracted from the making process and Claustra begins to appear to be the outcome of an antecedent concept or idea. ${ }^{4}$

Whereas the sculpting process undoubtedly involves considerable mental activity it does not follow that the locus of creativity is cerebral. The above description suggests not only that the creativity has an extracorporeal spatial coordinate but that its locus goes beyond the dimension of space. It exists in an experiential dimension in which time, space and creative change are reciprocally co-constructed. From a subjective point of view, space is not

\footnotetext{
${ }^{3}$ The artist experienced the sculptures-in-formation as though they had feelings and intentions. What we suggest this indicates is that the creative activity is being guided by environmental influences over which the artist feels he has limited agency.

${ }^{4}$ The point we would emphasise here is the contrast the artist is making between how the creative process feels in the presence of a dynamic material such as clay and how it appears in retrospect when the influence of the creative environment is no longer present.
} 
a pre-existing container, vacant until I fill it with a sculpture, subjective space is created along with the sculpture ${ }^{5}$.

\section{Haytor, the Vogelherd Lion (and the Jura)}

The most conspicuous geological feature of Dartmoor (Devon, UK) is its granite outcrops known as Tors. Sculpted by the erosion of wind and rain they take up extraordinary and beautiful forms. Haytor is a particular fine example (see Figure 3).

Insert Figure 3 here

One day, while sculpting, something about the shape of the clay reminded me of Haytor. Or rather, a memories of Haytor were influencing the emerging clay form while simultaneously being brought forth by the clay itself. As Høffding and Martigny (2016) point out, memories are not static records of the past like photos in an album. To bring to mind something from the past is to have a new, unique experience in the present. From the window of my workshop in Geneva I can see the Jura Mountains (see Figure 4) but I cannot see Haytor. By bringing up an image of the Tor on my computer I now had Haytor in front of me with the Jura beyond.

Insert Figure 4 here

Named after the cave in the Swabian Jura (Southwest Germany) in which it was found, the Vogelherd Lion is a small, Palaeolithic sculpture carved from mammoth ivory. On the windowsill of my workshop, between the image of Haytor and the Jura Mountains, stood a

\footnotetext{
${ }^{5}$ This paragraph expresses the central argument of our paper. It concerns whether creativity and problem-solving activity are understood and studied as emergent properties of the brain or as phenomenological constructs.
} 
replica of the Vogelherd Lion (see Figure 5) that I had ordered from Ebay some months earlier for similar reasons that I had brought up the photo of Haytor on my screen. Something in the work I had been doing at the time prompted a memory of it. Until the two (or three including the Jura Mountains in the background) were brought together within my gaze, I had not considered joining them in a project. Now in an "aha!" moment I saw that Haytor and the lion had similar forms and yet, by transposing one upon the other something new could emerge. While this appears to be an example of insight, given that it took place out there, in plain view, it is perhaps better described as "outsight"'

Insert Figure 5 here

\section{The Jali Screen}

In 2013 I made a large ceramic sculpture called Extended Phenotype 4 (see Figure 6, left panel). To lend support during construction, I created an internal clay "scaffolding" which was hidden inside once the finished piece was fired and reconstructed (see Figure 6, right panel). The scaffolding was serious, efficient and workmanlike but it also had a touchingly naive aspect to it. The interplay between these two, conflicting impressions gave it both force and appeal which I was sorry to have to hide from view.

Insert Figure 6 here

\footnotetext{
${ }^{6}$ It is important to note that our use of the word "outsight" does not imply a reversal in the polarity of agency nor of intention. There is an interaction going on. The artist played his part in constructing the environment in which he works, placing artefacts on the windowsill, working while facing the mountains etc. And, of course, the direction of his gaze determines what he sees. Our use of the word "outsight" implies a greater flexibility about what we mean by "cognitive". Internalist models of insight equate processing with neural processing. We, along with Malafouris (2013), are proposing that environmental changes should be included as part of the process of cognition.
} 
The feeling of sorrow meant that, as the Claustra project began to take shape, it did so, perhaps, with the tacit acceptance that the scaffolding of Phenotype 4 would rematerialize to provide visible support to the "cut" walls of Claustra. As such, although I hoped that the juxtaposition of rock-like surface and naive-but-functional scaffolding would create a bizarre yet coherent tension, in reality, I had no idea what the two different formations would make of each other. I therefore made a small model of the entire structure not only to investigate the specific interplay between scaffolding and rock-face but also to think about the overall proportions and structure of the two pieces and the relation between them (see Figure 7).

Insert Figure 7 here

The function of the model was not to make manifest a foregoing internal image of an artwork. Nor was it intended as a miniature version of the final work any more than a map is a version of a particular territory. Like a map, a model helps to orient exploration. It pinpoints the location of some significant landmarks, while leaving the terrain in-between largely unknown. Making a model is an important transformative stage, creating certain constraints which, while not determinant, served to provide some structural stability. In so doing a model paradoxically creates the freedom to take risks and thus make discoveries that would be impossible in a completely unconstrained environment ${ }^{7}$.

With regard to exposing the relationship between scaffolding and rock-face, the model suggested a certain antagonism between the two which I can only describe in the following metaphorically animistic terms. "The well-executed stone surface took exception to the rudimentary and primitive construction of the scaffolding." I was surprised because it was

\footnotetext{
${ }^{7}$ Here the artist describes his use of a model in terms that are similar to Kirsh's notion of cognitive scaffolding. Unlike the rest of the artist's narrative the implicit notion of the model as a cognitive tool brings environmental change into the intentional dominion of the artist.
} 
exactly this juxtaposition that had appealed to me when looking at the relationship between the support system for Extended Phenotype 4 and the organic/mineral external body of the piece. To investigate further, I began the construction of a full-size model of two sections of the sculpture (see Figure 8).

Insert Figure 8 here

I constructed the matrix of the lower section in a reasonably precise manner before returning to a more naïve-but-functional construction for the upper section. Comparing the two I found that, unlike the naive version, the precisely made matrix suggested a rhythm, mystery and strange formality that lived in happy yet contradictory coexistence with the rock surface. I began the construction of the final, full-scale version in this careful and precise way. As I did so, the work brought to mind the perforated ornamental screens or 'Jali' that are found in Indian and Islamic architecture (Mashrabiya). As the word "latticework" replaced "scaffolding" as a description of my perception of the structure, the work began to create similar paradoxical separations that one finds in Mosques, cloisters or in the confession box (see Figure 9).

Insert Figure 9 here

I was aware of no prior intention to refer to Islamic or Indian architecture or to the confession box. The association between material structure and religious conceptualisation developed only as I manipulated the sculpture. Nonetheless, when an exciting act of creativity like this takes place between my hands, I am tempted to take the credit for it. This act of self-affirmation (referred to in social psychology as the "self-serving bias" e.g. 
Mezulis, Abramson, Hyde, \& Hankin, 2004) requires the development of a hylomorphic attitude in which agency becomes a property of the person rather than the system ${ }^{8}$.

\section{Rotating the Cut}

Quite early in the development of the project, I recalled seeing an installation many years before by Damien Hirst, Prodigal Son (1994). It presents the body of a dead calf, cut along its longitudinal axis, each half placed in a tank of formaldehyde with the two tanks positioned opposite each other. I cannot now remember whether it had been possible to walk in the space between the two tanks or whether I had merely thought that it would have been good if it had been possible. Either way, my recollection of Prodigal Son came with the conviction that my experience (real or invented) of being between the two tanks must have been instrumental in the genesis of Claustra. But this assumption flew in the face of the order of events: it was the ongoing work on Claustra that brought Prodigal Son to mind, not visaversa. I have written elsewhere (March, 2017) about this paradoxical phenomenon whereby elements that are felt to have inspired a work are not present at its conception, but emerge instead during the course of its development. It is as though the emerging work brings its own antecedents into being. Normally when this happens, it is swiftly followed by a reexperiencing of the genesis of the work, this time with its origins in their so-called proper, chronological order. Despite the sense of history re-writing itself, I suspect that the reordering of experience has less to do with putting things in the correct order and more to do with the influence of a pervasive ontological view predicated upon cause and effect. Within this view, effect never precedes cause ${ }^{9}$.

\footnotetext{
${ }^{8}$ The artist describes two events that surprise him: first, the lack of coherence between the rock formation and naïve scaffolding and second, the reference to religious architecture. Surprise suggests an unexpected turn of events which in term suggests a predictive failure. We can understand this in terms of the artists's dual role as participant-observer and a relative move away from participation towards observation.

${ }^{9}$ The point here is that the notion of causative antecedents has little place in a cognitive system that includes a material environment because the structure of influence is, by definition circular rather than linear. The process that Malafouris (2013) calls "Enactive Signification" adroitly captures the formation of instantaneous circles of
} 
Hirst positions the two cut sides of Prodigal Son facing each other. When the installation came to mind in the context of Claustra, the thought included the implicit assumption that Claustra too would be oriented in the same fashion. However, in order to build the 'rock' sides of Claustra in a symmetrical manner, it was necessary to position those two sides facing each other so that I could see and have access to both simultaneously. This arrangement encouraged me to work in an unexpected way. After having initially built up the rough shape of each side separately (in series) I began to work on both sides simultaneously. Standing between the two sides, astride the axis of symmetry, I would place each hand on equivalent parts of the two rock faces and sculpt use mirrored gestures, often with my eyes shut, in order to better feel the symmetry through my fingers.

Symmetry was not something that was imposed. It developed in the following way. One side (A) might look and feel more appealing than the other (B). In response, I would concentrate on $\mathrm{B}$, bringing it closer to the form of A. But during its transformation, B might take on a form that went beyond that of A. In response, returning to A, I would try to steer it towards B and so on: the two sides of the installation nudging each other backwards and forwards towards their final composition - each serving as a dynamic reference for the other. As such, my role was not one of instigator or initiator. Rather, I was part of a decision making body that included the ever changing morphology of the clay. As with the lump of clay I mentioned earlier that referred to Haytor, the morphology of Claustra makes references to other things. But this is not the same as representing other things. The creative system that I am calling Claustra, and which included the actions of my body, brought itself about. It is a re-presentation neither of an existing structure nor of a pre-conception.

Once the two halves were complete I left them with the rock surfaces facing each other while they dried. I am not sure at what point this orientation came to be that of the final 
installation in the exhibition. The two structures had spent several months growing and developing in this position and I had spent much of that time between the two of them with my hands upon both. This left the thought of rotating the two halves to bring them into line with Prodigal Son difficult to entertain. Of course, once the sections were fired I could physically compare the two options. Doing so left me in no doubt that the exhibition positioning should follow the construction orientation.

\section{Conclusions}

It might be argued that our suggestion to replace "insight" with "outsight" as a description of a moment of problem resolution or of creative discovery does nothing more than change the direction of a linear model of causation from one that formerly had mental activity determining external activity to the reverse. We hope that it is clear both from the experimental material we have presented as well as the case study that this was not our intention. Rather, we have put forward a systemic, interactional model in which the question of the origin of causation becomes irrelevant. By concentrating on the process of creative problem-solving in relation to the manipulation of material things we do not wish to minimise the importance of cerebral activity. Rather, by redefining "outsight" and promoting it as an alternative to "insight" we wish to draw attention to the fact that real-world problem solving can never be properly understood by divorcing it from the real world within which it not only occurs but also has a hand in creating and influencing.

To achieve this, we have taken a two-pronged approach. First, by reconfiguring the traditional insight problem solving research procedure with physical models of the problem, we illustrate how eagerly participants embrace the opportunity to use artefacts to resolve problems successfully that were beyond the scope of their internal resources alone. We are not claiming that humans have no capacity for mental simulation nor that it cannot be used in a problem-solving task. Clearly second order problem solving, based on abstract representations of the world, is not only possible but is routinely encountered in laboratory 
environments. In addition, much psychometric research in occupational psychology profiles people's ability to solve problems this way, with important employability implications. Instead, we argue, along with Clarke (2010), that participants engage in isolated mental activity only if they are prevented from being in real-time relation with the world. Of course, one might argue that by changing, for example, the socks riddle into a physical model we are simply removing a tricky problem and replacing it with a simple sorting task. But that which is unknown remains the same, and rather than simulating different permutations and deriving the consequences of misleading odds information, the problem solver simply gets to work, with the world, to arrive at a satisfying answer. It is the coupling with the world that enhances creative problem solving.

A reviewer pointed out that some insight problem solving research under laboratory conditions employ problems that might be candidates for first order problem solving as we define it here. The 9-dot problem is a case in point. This is an interesting problem although important elements of that problem remain static. The 9-dot problem seems to us to be more first order but not completely first order. Partly because the page on which it exists can be both a real space and a virtual space so the domain of what is part of the problem and what lies beyond it is ambiguous. More important, the fact that it is difficult to solve is not evidence against the advantages of being able to interact with a model. It is a difficult problem which would be made even more difficult if it was turned into a pure second order task (imagining the 9 dots and the lines through them or preventing participants from using their hands or fingers to trace imaginary patterns). More generally, interactivity is not a panacea, and some problems however reified into a physical model, remain difficult to solve. There is also research that illustrates how the impact of interactivity is moderated by individual differences: For example, basic arithmetic skills, working memory capacity or mathematics anxiety moderate the impact of interactivity on mental arithmetic performance (e.g., Guthrie \& Vallée-Tourangeau, 2018). What is important to note, though, is that when 
second order problems are turned into first order problems (by offering participants the opportunity to manipulate and transform a physical model of the problem), problem solving performance is dramatically transformed (for both analytic and so-called insight problems). In addition to the illustration offered in the current manuscript with the 17 animals problem, this was demonstrated with matchstick algebra problems (Weller, Villejoubert \& ValleeTourangeau, 2011) and the cheap necklace problem (Henok, Vallée-Tourangeau \& ValléeTourangeau, 2018). In Fleck and Weisberg (2013), insight problems presented with material artefacts are solved differently from those without, although this is an aspect of their procedure that draws no commentary from these researchers (Vallée-Tourangeau, 2014). The scaffolding properties of interactivity have also been established for a range of transformation or analytic problems, including river crossing (Guthrie, Vallee-Tourangeau, ValleeTourangeau, \& Howard, 2015), Luchins's water jars (Vallee-Tourangeau, Euden, \& Hearn, 2011), mental arithmetic (Vallée-Tourangeau, 2013), Bayesian reasoning (ValléeTourangeau, Abadie, \& Vallée-Tourangeau, 2015), and anagrams (Ross \& ValléeTourangeau, under review).

It is also worth noting that the pioneering work on the psychology of insight problem solving in the first few decades of the 20th Century involved participants (and in the case of Köhler's ethnographic research, chimpanzees) working on problems that were presented with various objects and involved physical interaction. However, neither Duncker nor Maier emphasised the constitutive role of interactivity in the genesis of the solution. Duncker (1945) investigated functional fixedness and compared solution rates between conditions involving pre-utilization (boxes filled with other objects, such as matches) or without preutilization (empty boxes). Duncker's analysis did not focus on actions leading to a solution and his theoretical account was formulated to offer a sharp contrast with behaviourist and associative models of problem solving. Having said this, there are passages in section 8 of Duncker (1945) where he discusses the importance of "varied commerce with things" and 
reports a series of "stick experiments with infants" (pp. 69-71) to "illustrate the importance of varied commerce with things and situations for problem solving" (p. 71). The stick was used to retrieve 'some attractive object' only after a series of chance contacts between the stick and the object. Thus, some goal-driven behaviour in infants evolves from experiencing the positive results of random movements. In Maier's (1931) paper on the two-string problem, his research focus was on hints, not on how interactivity distilled a solution.

In the second section of our binary approach, we offer a detailed demonstration of how creative decision making can be seen to be embedded in the process of material change by presenting a subjective account from an artist in the process of an extended creative engagement. In this account we suggest that the focus on symmetry becomes deconceptualized by considering it in its historical context. In addition, the recursive relationship between the process of finding symmetry and of the final morphology undermines explanations of the creative process that describe art as serving a representative role for something that lies beyond the system of production.

Likewise, descriptions of the transposition of Haytor and the Vogelherd Lion and of the evolution of scaffolding into latticework both undermine explanations of creation that are couched in terms of insight. It is our contention that an appreciation of the decision-making process in its physical and historical context provides a strong argument for the importance of a reciprocal relationship with material in relation to artistic practice.

The credibility of our argument depends upon the status of the subjective account from which it is drawn. As mentioned earlier, subjective accounts by definition do not provide objectively verifiable material. Despite this, we think such accounts have much to offer for creativity research. This is not the place to present an exhaustive review of the place of subjectivity in the study of creativity and so we will limit ourselves to four points.

(1) The case-study narrative embeds creative change in a series of step-wise, externally observable, cognitive-environmental transformations. Although the artist, Paul March 
believes that the process of artistic creation occurred in the course of these steps, it is not necessary to accept that the artist's beliefs are correct in every detail. We seek only to establish that it is plausible that the phenomenon called insight might be better understood by looking for similar step-wise shifts in the cognitive-environmental architecture of a problemsolving situation. Thus, it is not the specific steps that are crucial but the nature of the transformative process itself that we wish to highlight. In Pandora's Hope Latour (1999, chapters 4 and 5) uses a case study to explore, reveal and describe the nature of the scientific process. In our description of the artistic process we proceed in an analogous way to Latour's description of Pasteur's work on lactic fermentation. Like Latour, by taking a step-by-step approach and by anchoring each step in material transformation, we hope to avoid the situation in which objective and subjective accounts find themselves in opposition. Like the argument made by Latour, the approach is not objective in the sense that it provides a scientifically valid outcome, but it is empirical in the sense that the account is constructed around the existence of observable artefacts (drawings, models, photos etc.). The reader can check the artist's interpretations by referring to one of more of the images of these artefacts.

(2) We wholeheartedly agree with the endeavour to provide an objective account of the process of creativity and its appreciation. Indeed, the first half of the paper attempts to do just that. But creativity is also a subjective and experiential activity. The materially-embedded phenomenological approach that we use in the second half of the paper is one attempt to capture something of the creative experience itself. We are not claiming that a phenomenological account is better than an experimental account. We see the two as complementary. One seeks validity whereas the other captures and highlights aspects of creative change that may be worthy of future exploration or even validation. Finally, within the domain of qualitative research, the sort of analysis that can be provided by a first-person account is of a different order from those studies that have interviewed artists about the creative process. Once again, we are not suggesting that one is better than the other. Rather, 
by reading this account alongside, for example, the recent paper by Sawyer (2018, in this journal) we can begin to understand the extent to which artists are using art-making as a meta-activity, that is, as a way of understanding the process of art-making itself. Of course, even if this is true of some artistic activity, there is no reason to believe that it is true of all of the arts, let alone creative activity in general. What we think this case study does do is to highlight the notion that the process of creation has the potential for being a self-conscious endeavour or a meta-activity. By this we mean that art-making has the capacity to investigate its own process. Indeed, within art history circles, this self-consciousness is seen as one of the principle ways in which the modernist movement separated itself from classical art (e.g., Hughes, 1980). Thus, not only is this meta-creativity important to study as part of the creative act, the study of creation itself will be greatly enhanced by collaborating more with working artists.

(3) The case study is constructed around the emergence of a series of material artefacts. The narrative respects and follows the chronology of these appearances. The commentary by the artist is temporally linked with each artefact and his reports of cognitive states are likewise linked either to the material transformation within an artefact or across one or more artefacts. These descriptions should not therefore be misconstrued as introspections, making reference to the unverifiable workings of an inner mind. Quite the reverse, they are comments concerning the processes of an extended and visible mind at work. By providing images of the various artefacts together with a description of how they are related, the reader has the evidence to make a judgement, not about whether the story is true, but about whether the story can account for the facts. In our view the story describes the process of creation with a parsimony and transparency that is simply not available to descriptions that are based on the workings of an internal mind. A similar approach has been developed by within the field of Postphenomenology by Rosenberger and Verbeek (2015; see below). 
(4) The use of artefacts to support or scaffold an argument or interpretation is routinely used in other disciplines, namely Archaeology and Anthropology. In particular, within the domain of material culture, the study of the behaviour of things in relation to people is central to understanding both the development of culture (see Lucas 2010 for an overview) and of cognition (see for example the Material Engagement Theory of Malafouris, 2013). In addition, over the last 25 years, a phenomenological approach to the study of material culture has developed (see Thomas, 2006, for an overview)

From a philosophical perspective, Idhe (2009) has developed Heidegger's ontological position into a postphenomenological account of the humans-technology interface. Ihde and others (e.g. Rosenberger and Verbeek, 2015) have developed a case-study methodology based upon first-person accounts and self-conducted studies in order to understand how technology and humans co-construct subjective experience. Rosenberger and Verbeek (2015) describe the postphenomenological method as "empirical philosophy": by which they mean that, by focusing on the co-constructed relationship between human and technology, they can transform the metaphysical into something physical and thus available for empirical investigation.

There is no denying that the approaches that have developed from material culture and from phenomenology take a radically different ontological position from the one traditionally held within the cognitive sciences. For example, Postphenomenology and Material Engagement Theory both argue that the human-world relationship is one in which mind and material are ontologically linked in a relational, recursive and creative ecology. Second, both emphasize the importance of the role of practice and experience over that of internal representations (Ihde and Malafouris, 2018). It seems to us that the developing distributed cognition movement within the cognitive sciences has much in common with the material culture approach. Our motivation is to create and develop multidisciplinary links between 
scholars working in different fields, with different methodologies but with the same ontological allegiances. 


\section{References}

Ansburg, P.I., \& Dominowski, R.L. (2000). Promoting insightful problem solving. Journal of Creative Behavior, 34, 30-60.

Boden, M.A. (2004). The creative mind: myths and mechanisms. London: Routledge.

Bowden, E., \& Grunewald, K. (2018). Whose insight is it anyway? In F. Vallée-Tourangeau (Ed.), Insight: On the origins of new ideas (pp. 28-50). London: Routledge.

Cacioppo, J. T., Petty, R. E., \& Kao, C. F. (1984). The efficient assessment of need for cognition. Journal of Personality Assessment, 48, 306-307.

Casati, R., \& Varzi, A. C. (1994). Holes and other superficialities. Cambridge, MA: MIT Press.

Chuderski, A., \& Jastrzębski, J. (2018). Much ado about aha!: Insight problem solving is strongly related to working memory capacity and reasoning ability. Journal of Experimental Psychology: General, 147, 257-281.

Clark, A. (2010). Material surrogacy and the supernatural: Reflections on the role of artefacts in "off-line" cognition. In L. Malafouris \& C. Renfrew (Eds.), The cognitive life to things: Recasting the boundaries of the mind (pp. 23-28). Cambridge: McDonald Institute Monographs.

Fleck, J. I., \& Weisberg, R. W. (2013). Insight versus analysis: Evidence for diverse methods in problem solving. Journal of Cognitive Psychology 25, 436-463.

Gallagher, S. (2018). Decentering the brain: Embodied cognition and the critique of neurocentrism and narrow-minded philosophy of mind. Constructivist Foundations, 14: 008.

Gilhooly, K. J., \& Fioratou, E. (2009). Executive functions in insight versus non-insight problem solving: An individual differences approach. Thinking and Reasoning, 15, 355-376. 
Gilhooly, K., \& Webb, M. E. (2018). Working memory and insight problem solving. In F. Vallée-Tourangeau (Ed.), Insight: On the origins of new ideas (pp. 105-119). London: Routledge.

Guthrie, L. G., \& Vallée-Tourangeau, F. (2018). Numbers in action: Individual differences and interactivity in mental arithmetic. Cognitive Processing, 19, 317-326.

Guthrie, L. G., Vallée-Tourangeau, F., Vallée-Tourangeau, G., \& Howard, C. (2015). Learning and interactivity in a transformation problem. Memory \& Cognition, 43, 723735.

Haran, U., Ritov, I., \& Mellers, B. A. (2013). The role of actively open-minded thinking in information acquisition, accuracy, and calibration. Judgment and Decision Making, 8, 188-201.

Henok, N., Vallée-Tourangeau, F., \& Vallée-Tourangeau, G. (2018). Incubation and interactivity in insight problem solving. Psychological Research (online first).

Hughes, R. (1980). The shock of the new: Art and the century of change. London: Thames and Hudson.

Hutchins, E. (1995). Cognition in the wild. Cambridge MA: MIT Press.

Hutchins, E. (2010). Cognitive ecology. Topics in Cognitive Science, 2, 705-715.

Høffding. S \& Martiny. K., (2016) Framing a phenomenological interview: what, why and how. Phenomenology and the Cognitive Sciences, 15, 539-564.

Ihde, D. (2009). Postphenomenology and technoscience: the Peking University lectures. New York.

Idhe, D \& Malafouris, L. (2018). Homo Faber Revisited: Postphenomenology and Material Engagement Theory. Philosophy \& Technology.

Köhler, W. (1925/1957). The mentality of apes. London: Penguin Books. 
Kirsh, D. (2009). Problem solving and situated cognition. In, P. Robbins \& M. Aydede (Eds.), The Cambridge handbook of situated cognition (pp. 264-306). Cambridge: Cambridge University Press.

Latour, B. (1999). Pandora's hope: Essays on the reality of science studies. Cambridge MA: Harvard University Press.

Lave, J. (1988). Cognition in practice: Mind, mathematics and culture in everyday life. New York: Cambridge University Press.

Lucas, G. (2010). Fieldwork and collecting. In D. Hicks \& M. C. Beaudry (Eds.), The Oxford handbook of material culture studies. (pp. 43-59) Oxford: Oxford University Press.

Luo, J., \& Niki, K. (2003). Function of hippocampus in "insight” of problem solving. Hippocampus 13, 316-323.

March, P. L. (2017). Playing with clay and the uncertainty of agency: A Material Engagement Theory perspective. Phenomenology and the Cognitive Sciences. Malafouris, L. (2013). How things shape the mind: A theory of material engagement. Cambridge MA: MIT Press.

Mezulis, A. H., Abramson, L. Y., Hyde, J. S., \& Hankin, B. L. (2004). Is there a universal positivity bias in attributions? A meta-analytic review of individual, developmental, and cultural differences in the self-serving attributional bias. Psychological Bulletin, $130,711-747$.

Milner, M. (1950). On not being able to paint. Madison: International Universities Press.

Oberauer, K., Schulze, R., Wilhelm, O., \& Süß, H.-M. (2005). Working memory and intelligence-their correlation and their relation: Comment on Ackerman, Beier, and Boyle (2005). Psychological Bulletin, 131, 61-65.

Ohlsson, S. (2018). The dialectic between routine and creative cognition. In F. ValléeTourangeau (Ed.), Insight: On the origins of new ideas (pp. 8-27). London: Routledge. Rosenberger, R., \& Verbeek, P. P. (2015). A field guide to Postphenomenology. In R. 
Rosenberger, \& P-P. Verbeek (Eds.), Postphenomenological investigations: Essays on human-technology. (pp.9-41) Lanham, Maryland: Lexington Books.

Ross, W., \& Vallée-Tourangeau, F. (under review). Unknitting the meshwork: Interactivity, serendipity and individual differences in a word production task.

Steffensen, S. V., Vallée-Tourangeau, F., \& Vallée-Tourangeau, G. (2016). Cognitive events in a problem-solving task: Qualitative methods for investigating interactivity in the 17 animals problem. Journal of Cognitive Psychology, 28, 79-105.

Thomas, J., (2006). Phenomenology and material culture. In: C. Tilley, W. Keane, S. Kuchler, M. Rowlands, P. Spyer (Eds.), Handbook of material culture (pp. 43-59). London: Sage.

Vallée-Tourangeau, F. (2013). Interactivity, efficiency, and individual differences in mental arithmetic. Experimental Psychology, 60, 302-311.

Vallée-Tourangeau, F., Sirota, M., \& Vallée-Tourangeau, G. (2016). Interactivity mitigates the impact of working memory depletion on mental arithmetic performance. Cognitive Research: Principles and Implications, 1, 26.

Vallée-Tourangeau, F., Steffensen, S. V., Vallée-Tourangeau, G., \& Sirota, M. (2016). Insight with hands and things. Acta Psychologica, 170, 195-205.

Vallée-Tourangeau, G., \& Vallée-Tourangeau, F. (2014). The spatio-temporal dynamics of systemic thinking. Cybernetics and Human Knowing, 21, 113-127.

Weisberg, R., \& Suls, J. M. (1973). An information-processing model of Duncker's Candle Problem. Cognitive Psychology, 4, 255-276.

Weisberg, R. W. (2018). Insight, problem solving, and creativity: An integration of findings. In F. Vallée-Tourangeau (Ed.), Insight: On the origins of new ideas (pp. 191-215). London: Routledge.

Weller, A., Villejoubert, G., Vallée-Tourangeau, F. (2011). Interactive insight problem solving. Thinking \& Reasoning, 17, 429-439. 
Wilson, R. A., \& Clark, A. (2009). How to situate cognition. letting nature take its course. In P. Robbins \& M. Aydede (Eds.), The Cambridge handbook of situated cognition (pp. 55-77). Cambridge: Cambridge University Press. 
Figure 1. Claustra; stoneware 1.8 x $2.0 \times 1.8$ m, 2015 .

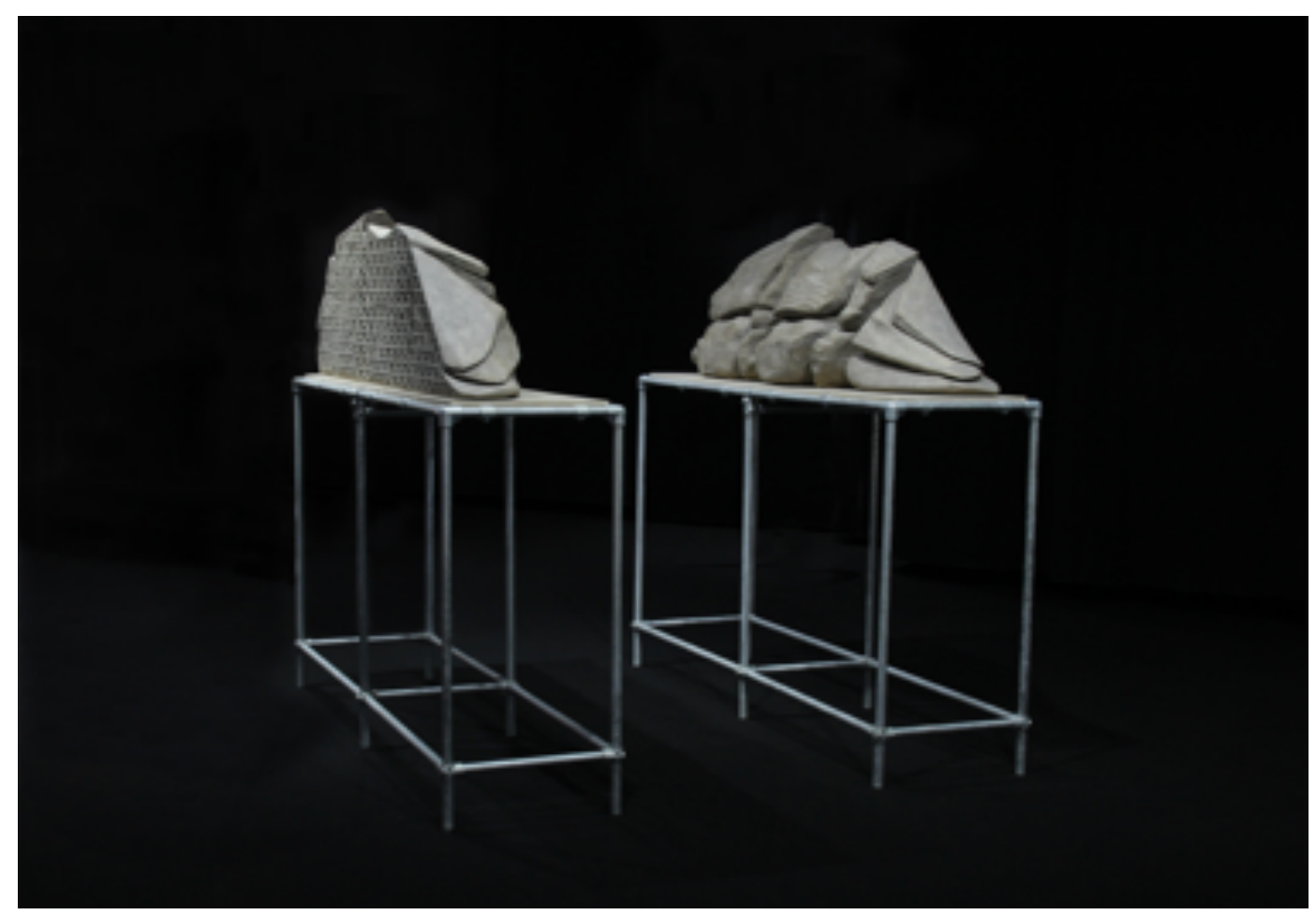


Figure 2. Substantia Innominata, III stoneware 0.3 x 0.4 x 0.2 m, 2014. 
Figure 3. Haytor, Dartmoor, United Kingdom.

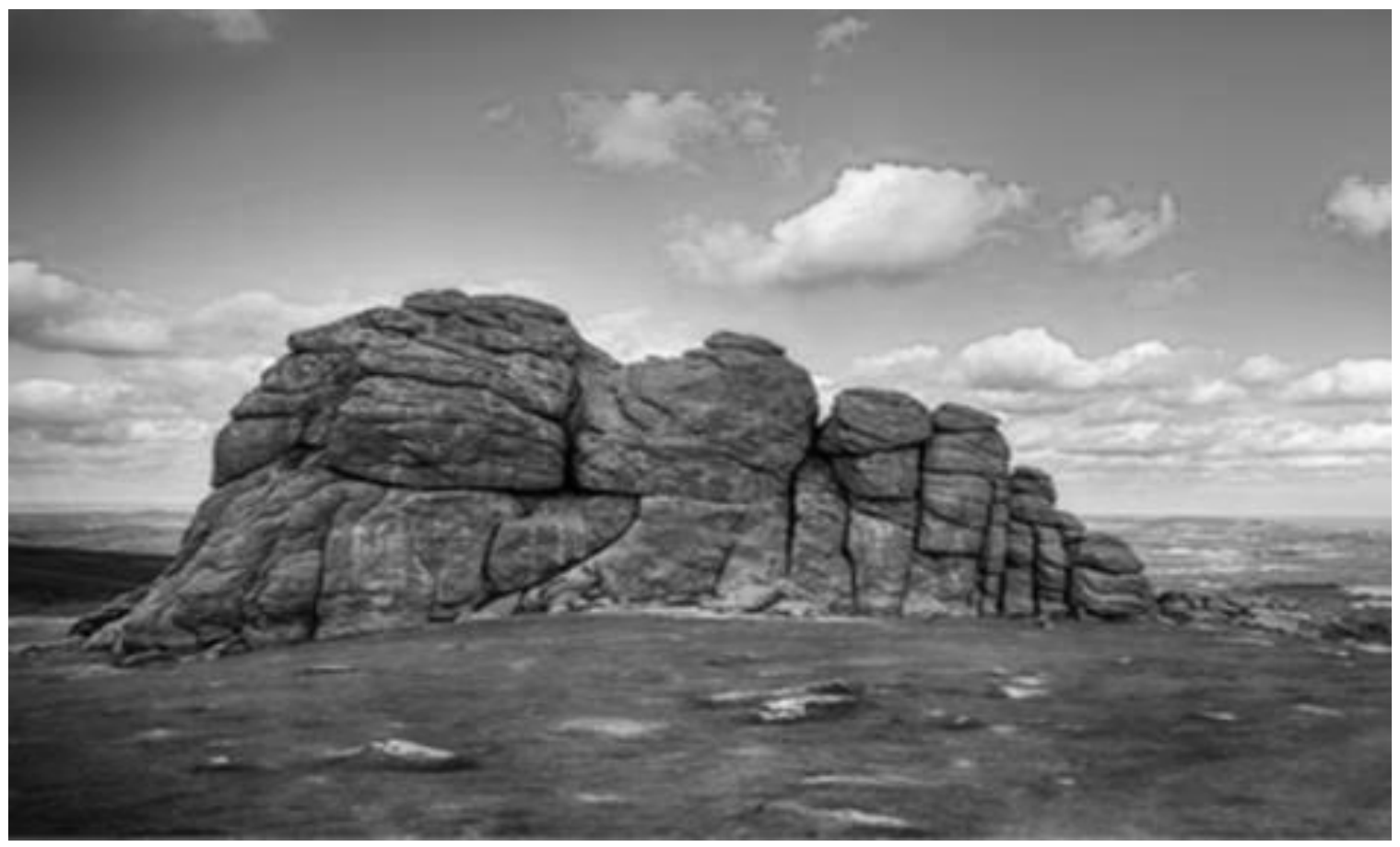


Figure 4. View of the Jura from the artist's workshop window.

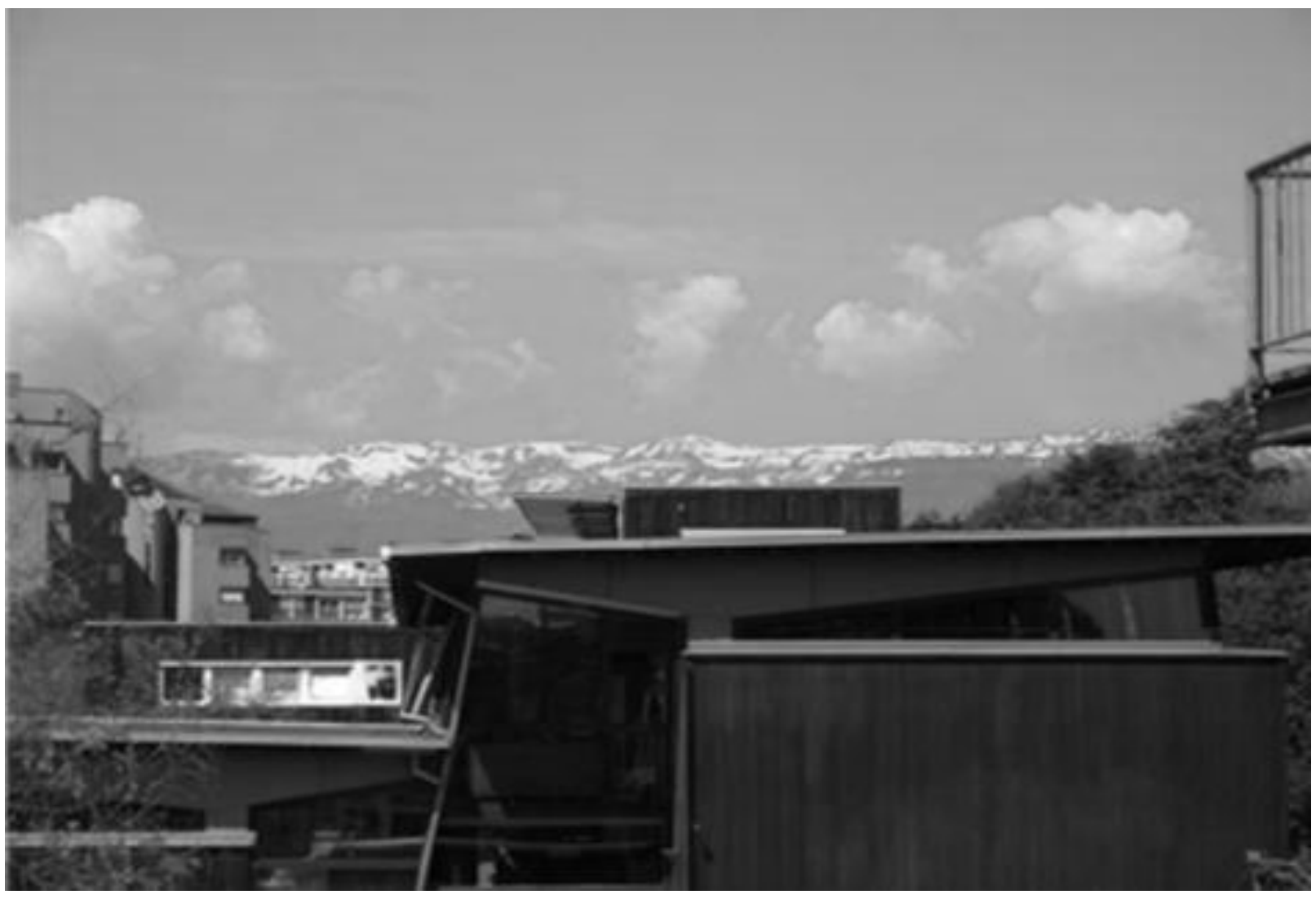


Figure 5. The Vogelherd Lion (left panel) and the replica sitting on the artist's windowsill (right panel).
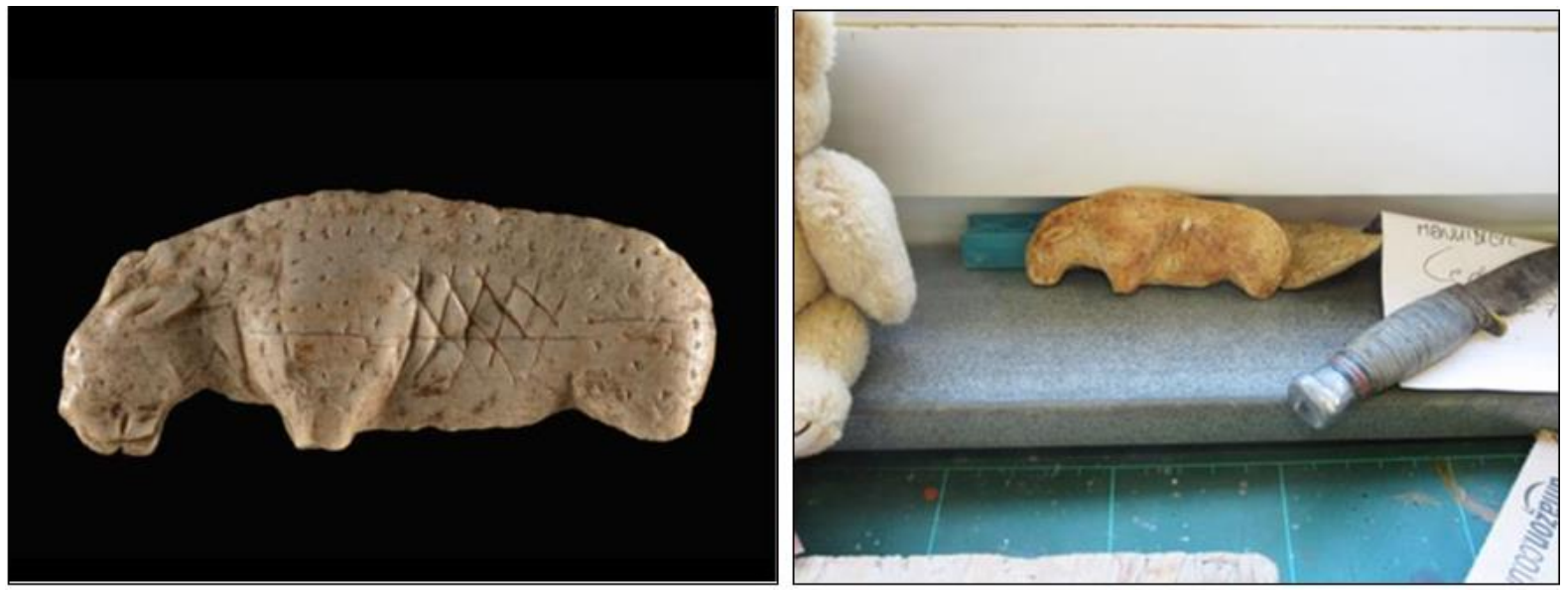
Figure 6. Extended Phenotype (extant) stoneware, $3.5 \times 1.0 \times 1.0 \mathrm{~m}, 2013$ (left panel); view of the inside of a section of Extended Phenotype 4 (right panel).
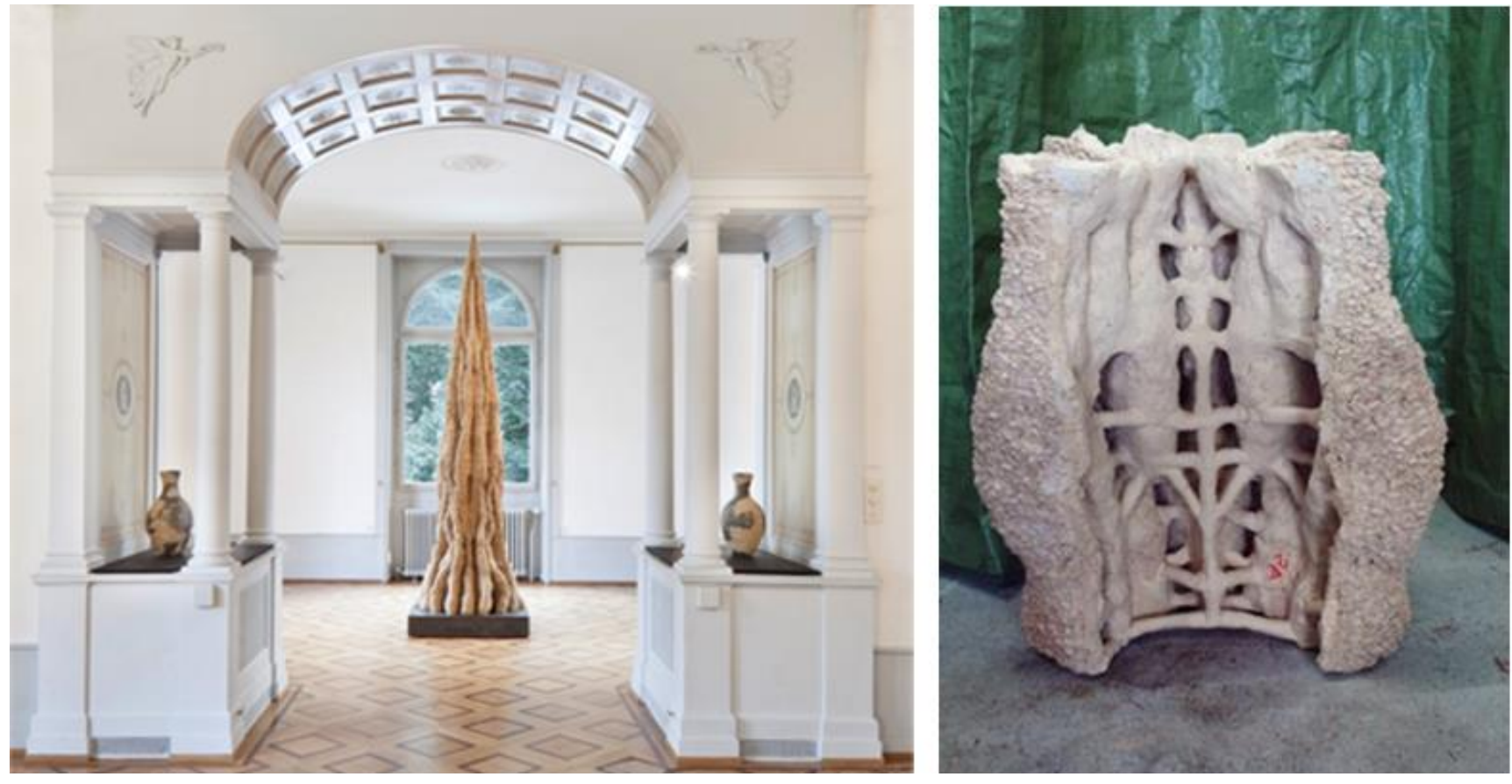
Figure 7. Model of Claustra. 0.22 x 0.15 x $0.08 \mathrm{~m}, 2015$

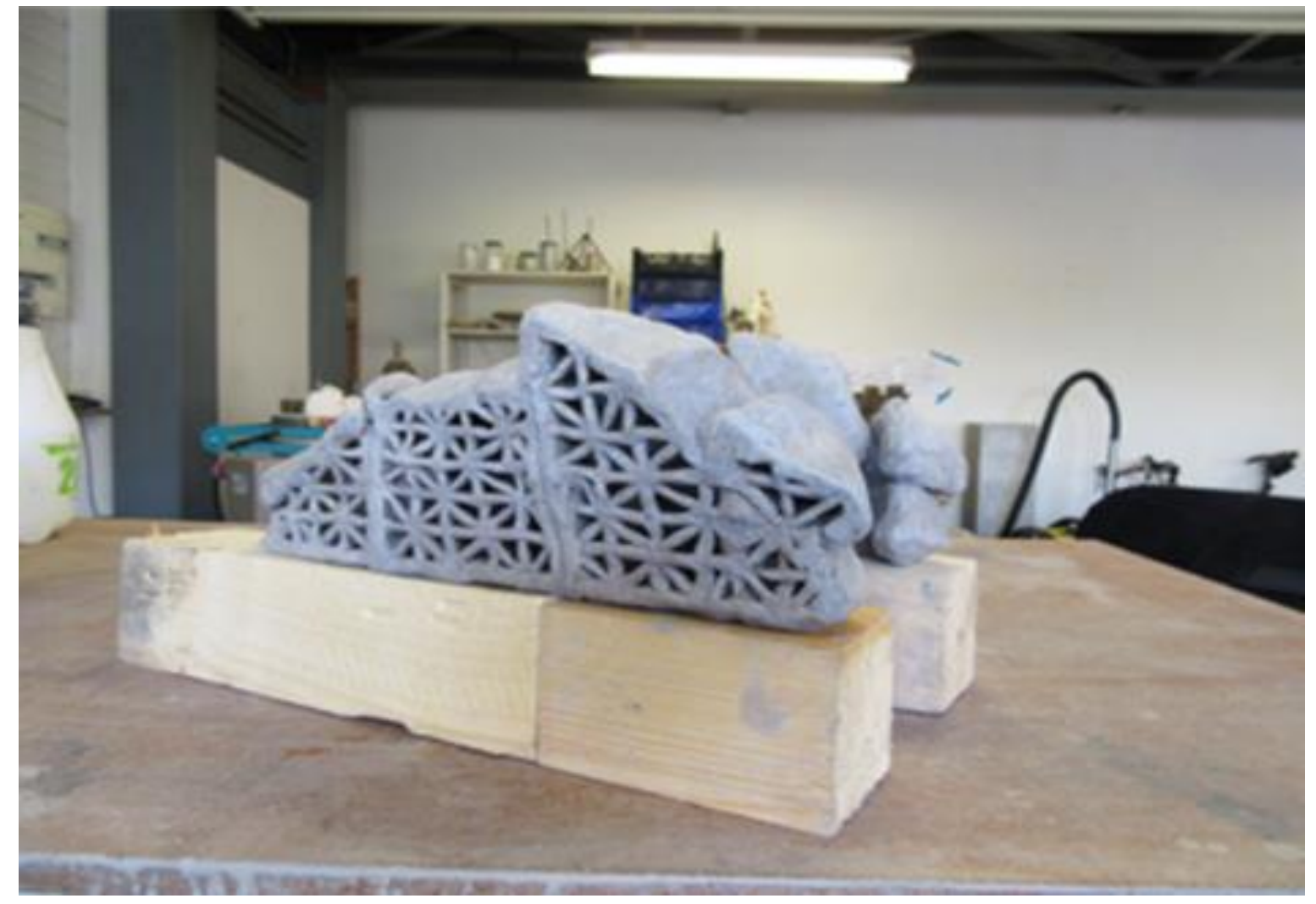


Figure 8. Two test sections for Claustra.

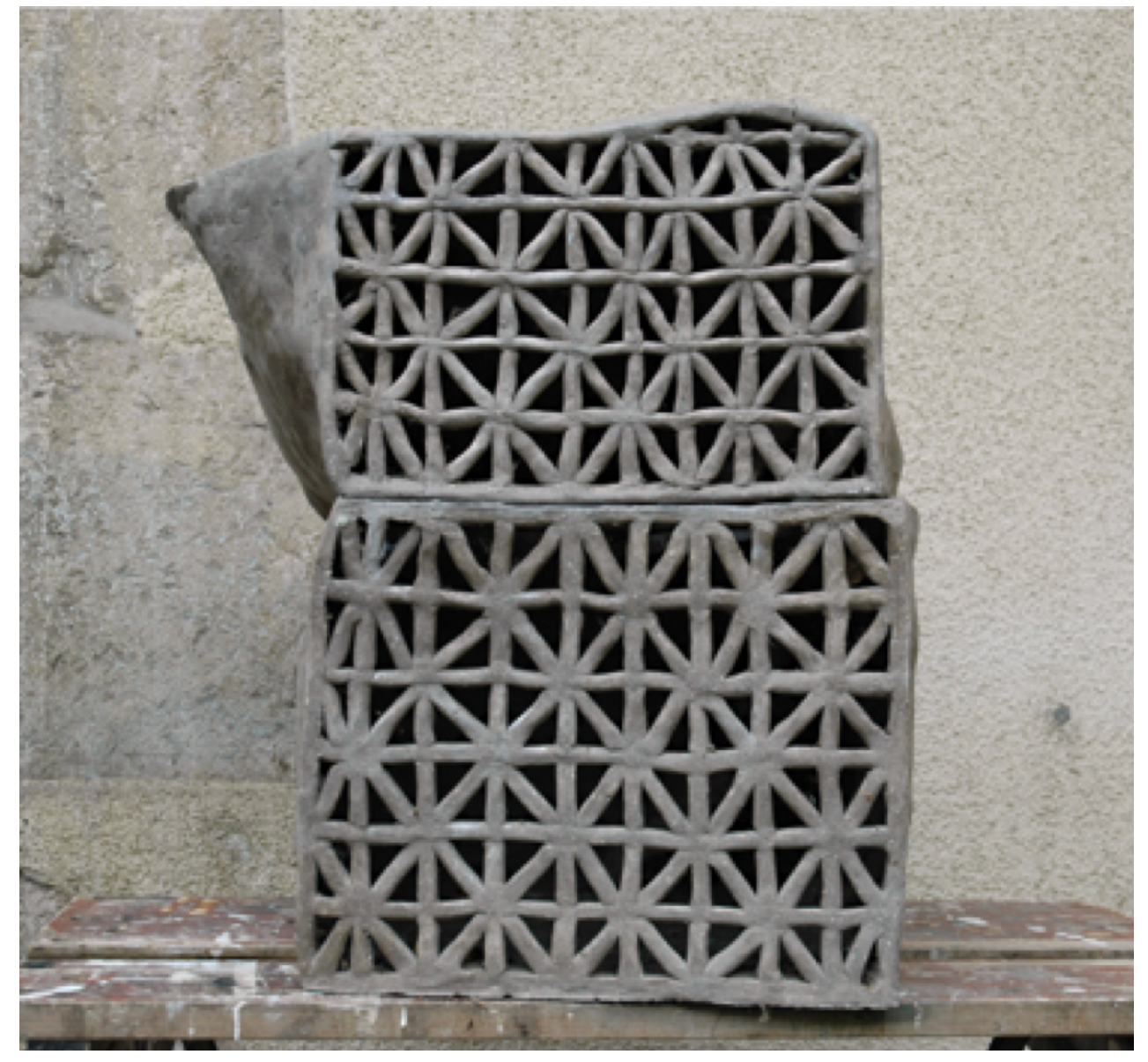


Figure 9. Example of Mashrabiya from the Alijaferia Palace, Zaragotha, Spain (left panel); view of the lattice work of Claustra (right panel).
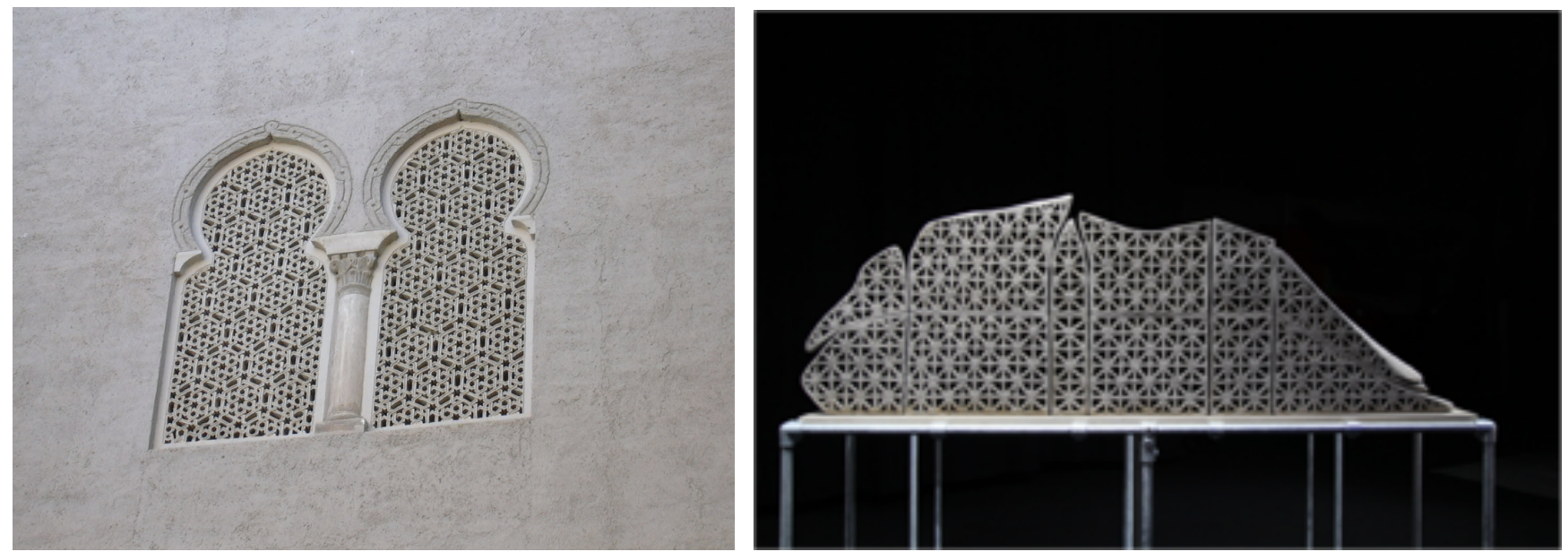